\title{
ENFERMAGEM DO TRABALHO EM PORTUGAL: CONTEXTO E PERSPETIVAS
}

\section{OCCUPATIONAL NURSING IN PORTUGAL: CONTEXT AND PERSPECTIVES}

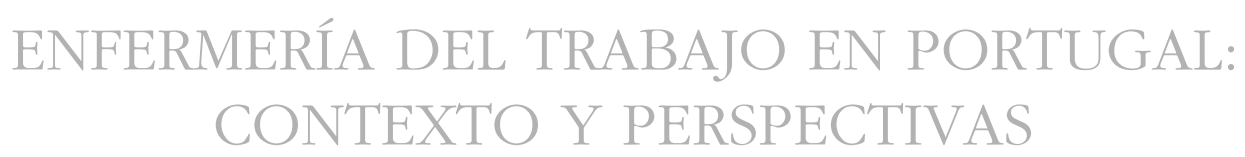

\author{
Vitor Manuel Teixeira Machado ${ }^{1}$ \\ Maria João Filomena dos Santos Pinto Monteiro ${ }^{2}$ \\ Maria Helena de Oliveira Penaforte ${ }^{3}$ \\ Vitor Manuel Costa Pereira Rodrigues ${ }^{4}$
}

Como citar este artigo: Machado VMT, Monteiro MJFSP, Penaforte MHO, Rodrigues VMCP. Enfermagem do Trabalho em Portugal: contexto e perspetivas. Rev baiana enferm. 2022;36:e45605.

\begin{abstract}
Objetivo: conhecer a perceção dos enfermeiros do trabalho no que respeita às áreas de conhecimento e intervenção. Método: estudo quantitativo, descritivo e transversal. Envolveu amostra de 472 enfermeiros do trabalho. Utilizou-se como instrumento de recolha de dados, que decorreu entre maio e setembro de 2017, questionário, alicerçado nas áreas nucleares de conhecimentos e competências do enfermeiro do trabalho e na escala de perceção das áreas de conhecimento e intervenção do enfermeiro do trabalho. Resultados: a Enfermagem do Trabalho foi entendida pela maioria como atividade profissional transitória, pelo facto de ser exercida como complemento financeiro à atividade principal ou porque não conseguiram colocação na área de exercício profissional da sua preferência. Conclusão: o exercício profissional nas áreas de conhecimento e intervenção foi desvalorizado ou não reconhecido e encarado pela maioria dos enfermeiros como transitório, pelo facto de quase metade ambicionar trabalhar no futuro nessa área, mas apenas em regime de tempo parcial.
\end{abstract}

Descritores: Enfermagem do Trabalho. Perceção. Educação Continuada. Assistência Integral à Saúde. Desenvolvimento de Programas.

Objective: to know the perception of nurses at work regarding the areas of knowledge and intervention. Method: quantitative, descriptive and cross-sectional study. It involved a sample of 472 occupational nurses. A questionnaire was used as a data collection instrument, which took place between May and September 2017, based on the core areas of knowledge and competencies of the occupational nurse and on the scale of perception of the areas of knowledge and intervention of the occupational nurse. Results: Occupational Nursing was understood by the majority as a transitory professional activity, because it was exercised as a financial complement to the main activity or because they could not be placed in the area of professional practice of their preference. Conclusion: the professional practice in the areas of knowledge and intervention was devalued or not recognized and seen by the majority of nurses as transitory, because almost half aspire to work in the future in this area, but only on a part-time basis.

Descriptors: Occupational Health Nursing. Perception. Education, Continuing. Comprehensive Health Care. Program Development.

\footnotetext{
Enfermeiro. Doutor em Ciências da Educação. Especialista em Enfermagem Comunitária. Centro Hospitalar de Trás-os-Montes e Alto Douro EPE. Chaves, Portugal. vito.machado@gmail.com. https://orcid.org/0000-0003-4388-2683.

Enfermeira. Doutora em Ciências da Educação. Professora do Centro de Investigação em Tecnologias e Serviços de Saúde, Escola Superior de Saúde, Universidade de Trás-os-Montes e Alto Douro. Vila Real, Portugal. https://orcid.org/0000-0003-06 I0-0670.

3 Enfermeira. Doutora em Enfermagem. Centro Hospitalar de Trás-os-Montes e Alto Douro EPE. Chaves, Portugal. https://orcid.org/0000-0002-4426-3545.

${ }^{4}$ Enfermeiro. Doutor em Ciências Biomédicas. Professor do Centro de Investigação em Desporto, Saúde e Desenvolvimento Humano, Escola Superior de Saúde,
} Universidade de Trás-os-Montes e Alto Douro. Vila Real, Portugal. https://orcid.org/0000-0002-2795-685X. 
Objetivo: conocer la percepción de las enfermeras en el trabajo con respecto a las áreas de conocimiento e intervención. Método: estudio cuantitativo, descriptivo y transversal. Involucró una muestra de 472 enfermeras de trabajo. Se utilizó un cuestionario como instrumento de recolección de datos, que tuvo lugar entre mayo y septiembre de 2017, basado en las áreas nucleares de conocimiento y competencias de la enfermera de trabajo y en la escala de percepción de las áreas de conocimiento e intervención de la enfermera de trabajo. Resultados: la Enfermería Ocupacional fue entendida por la mayoría como una actividad profesional transitoria, porque se ejercía como complemento económico de la actividad principal o porque no podían ubicarse en el área de práctica profesional de su preferencia. Conclusión: la práctica profesional en las áreas de conocimiento e intervención fue devaluada o no reconocida y vista por la mayoría de las enfermeras como transitoria, porque casi la mitad aspira a trabajar en el futuro en esta área, pero solo a tiempo parcial.

Descriptores: Enfermería del Trabajo. Percepción. Educación Continua. Atención Integral de Salud. Desarrollo de Programa.

\section{Introdução}

De acordo com a Agência Europeia para a Segurança e Saúde no Trabalho, apesar de o número de acidentes de trabalho ter diminuído cerca de $25 \%$ ao longo dos últimos dez anos, anualmente, as doenças relacionadas com o trabalho continuam a ser responsáveis, aproximadamente, por 2,4 milhões de mortes no mundo; destas, cerca de 200.000 ocorrem na Europa ${ }^{(1)}$.

Os enfermeiros são o maior grupo profissional da área da saúde que presta cuidados em contexto laboral à população ativa ${ }^{(2)}$, sendo, a enfermagem, vista como uma das profissões mais relevantes.

A Enfermagem do Trabalho é definida pela American Association of Occupational Health Nurses $^{(3)}$, como a prática especializada que oferece serviços e programas de segurança e saúde ao trabalhador, populações de trabalhadores e grupos da comunidade. Neste contexto de trabalho, são enfatizadas a promoção, proteção e recuperação da saúde dos trabalhadores. A sua prática centra-se na promoção e restauração da saúde, prevenção de doenças e lesões e ainda na proteção contra perigos ambientais. Estes enfermeiros combinam diversos conhecimentos que associam com perícia, equilibrando os requisitos, a fim de promover um ambiente de trabalho seguro e saudável.

Atualmente, em Portugal, a carreira de enfermagem está organizada por áreas de exercício e de cuidados de saúde que, entre outras, contempla a área de Enfermagem do Trabalho (art. 6. ${ }^{\circ}$ do Decreto-Lei n. ${ }^{\circ}$ 71/2019, de 27 de maio) ${ }^{(4)}$.
No entanto, apesar de estar integrada na legislação, não existe, em Portugal, a especialização em Enfermagem do Trabalho ${ }^{(5)}$.

A Direção Geral da Saúde, pela Orientação n. 009/2014, de 3 de junho ${ }^{(6)}$, considera a atividade do enfermeiro do trabalho como a que é dirigida à gestão da saúde do trabalhador ou de grupos de trabalhadores, com enfoque na promoção e proteção da saúde e bem-estar no local de trabalho, na prevenção de acidentes e doenças relacionadas ou agravadas pelo trabalho, com o propósito de promover ambientes de trabalho saudáveis e seguros. Esta autoridade de saúde, por meio dessa mesma orientação, pretendeu criar um registo de enfermeiros habilitados/autorizados a prestar cuidados de Enfermagem do Trabalho, estabelecendo os critérios e procedimentos necessários para o reconhecimento de habilitação e para a autorização "transitória” para o exercício.

Foi na década de 1940 que se fundou a primeira organização de enfermeiros do trabalho, a American Association of Occupational Health Nurses. Entretanto, nem o aparecimento desta organização nem os demais impulsos citados foram suficientes para que a Enfermagem do Trabalho ultrapassasse os obstáculos que, durante longas décadas, haviam de fazer parte da sua caracterização, como, por exemplo, a falta de uniformização de métodos, o trabalho isolado e a falta de formação específica ${ }^{(7)}$.

O bem-estar físico e o bem-estar emocional são duas dimensões inseparáveis da Enfermagem 
do Trabalho, uma vez que esta reside no estabelecimento da melhoria da saúde e da satisfação total dos trabalhadores em relação a si próprios, bem como na relação com os outros e na sua capacitação, para que conduzam, de forma eficaz, os processos de vida e de trabalho ${ }^{(8)}$.

Importa também referir que a Enfermagem do Trabalho pressupõe que os seus executores gozem de independência profissional durante o exercício das suas funções. Esse entendimento é alicerçado na garantia de que os enfermeiros do trabalho são dotados de competências profissionais que vão ao encontro das novas realidades da economia e são capazes de dar resposta às múltiplas exigências que caracterizam a maioria das organizações de trabalho do século $\mathrm{XXI}^{(9)}$.

Apesar dos problemas relacionados à sobrecarga de trabalho, aos ritmos de trabalho exigidos dos trabalhadores, aos objetivos de produção e às pressões rígidas para a máxima qualidade no menor espaço de tempo, entre tantos outros, que os trabalhadores atualmente enfrentam, não podem ficar de fora a visão integradora e favorecedora de ambientes de trabalho que a Enfermagem do Trabalho visa criar. Da mesma forma, a Enfermagem do Trabalho também não se pode mostrar indiferente à problemática dos conflitos interpessoais que se geram em muitas situações laborais, nem aos fenómenos de bullying ou à constante mutação tecnológica a que o trabalho está exposto $^{(10)}$.

Nesses cenários tão diferentes e, simultaneamente, distantes da realidade das unidades hospitalares, os profissionais da Enfermagem do Trabalho atuam. Essas circunstâncias colocam-nos, muitas vezes, em situações de conflito e de difícil solução. Na verdade, o enfermeiro do trabalho é um profissional de saúde que trabalha num palco em que os conflitos de interesse são protagonistas permanentes e obreiros do dilema: preservar e promover a saúde dos trabalhadores versus não lesar os objetivos de eficiência e produtividade da empresa ${ }^{(11)}$.

De acordo com o exposto, foi objetivo deste estudo conhecer a perceção dos enfermeiros do trabalho no que respeita às áreas de conhecimento e intervenção.

\section{Método}

Tratou-se de um estudo de natureza quantitativa, do tipo descritivo e transversal, realizado com 472 Enfermeiros do Trabalho. Foi utilizado um questionário constituído por duas partes: a primeira, continha 22 questões de caracterização da amostra; e a segunda, contemplando a escala de perceção das áreas de conhecimento e intervenção do enfermeiro do trabalho (EPACIET), era composta por 76 afirmações/itens, distribuídas por 8 áreas de conhecimento e intervenção ${ }^{(5)}$ : clínico, especialista, gestor, coordenador, consultor, educador para a saúde, conselheiro e investigador. Nesta última, era solicitado aos respondentes (enfermeiros) a valorização dada diante do seu posicionamento numa escala de concordância tipo Likert de 1 a 5 pontos, em que 1 correspondia a "discordo em absoluto" e 5, a "concordo em absoluto".

Este questionário de autopreenchimento, construído no Google Docs (https://goo.gl/forms/ eCUnaY4ZTZVLSK6L2), foi enviado aos Enfermeiros do Trabalho de Portugal. Nessa etapa, contou-se com a preciosa ajuda da Associação Nacional dos Enfermeiros do Trabalho e da Direção de Serviços de Prevenção da Doença e Promoção da Saúde da Direção Geral de Saúde, uma vez que essas instituições enviaram, por correio eletrónico, aos enfermeiros do trabalho considerados habilitados e/ou autorizados a exercer Enfermagem do Trabalho em Portugal, o link para o preenchimento do questionário. A recolha de dados decorreu entre maio e setembro de 2017. O critério de elegibilidade foi estar registrado na Associação Nacional dos Enfermeiros do Trabalho e na Direção de Serviços de Prevenção da Doença e Promoção da Saúde da Direção Geral de Saúde. O único critério de inclusão foi estar autorizado a exercer Enfermagem do Trabalho em Portugal.

Os enfermeiros que participaram do estudo foram informados e esclarecidos acerca dos objetivos, tendo sido assegurados o anonimato, a confidencialidade dos dados e a participação voluntária. Isto é, tratou-se unicamente de um estudo em que os indivíduos responderam voluntariamente a um questionário de autopreenchimento, 
construído no Google Docs, no qual constavam questões de caracterização e da escala de perceção das áreas de conhecimento e intervenção do enfermeiro do trabalho. Não existiram riscos nem desconfortos para os sujeitos que dele participaram. Salienta-se que, para o preenchimento on-line do questionário, era exigido: obtenção do consentimento informado e da sua participação voluntária. Para tanto, deveriam assinalar na "caixa" construída para o efeito, com carácter de obrigatoriedade, que, em caso negativo, inviabilizaria o respetivo preenchimento. Foi também facultada a identificação do investigador, como forma de possibilitar o contacto para esclarecimento de dúvidas e atestar a veracidade das intenções declaradas.

Quanto à análise e ao tratamento de dados, construiu-se uma base de dados no software informático Statistical Package for the Social Sciences (SPSS), versão 22.0, tendo-se recorrido à estatística descritiva (frequências absolutas, relativas e medidas de tendência central) e à aplicação de testes paramétricos ( $\mathrm{t}$ de Student), considerando-se um nível de significância de 5\%.

\section{Resultados}

Dentre os enfermeiros do trabalho inquiridos, 472 tinham idades compreendidas entre $22 \mathrm{e}$ 68 anos, sendo a média de idades de 38 anos; a maior percentagem de participantes (73,3\%) pertenceu ao género feminino (346). Dos participantes, 56,8\% possuíam o estado civil de casado/união de facto, 34,7\% eram solteiros e $8,5 \%$ eram divorciados ou separados. O grau de licenciado foi apresentado por 80,3\%, 18,9\% detinham grau de mestre, $0,6 \%$ o grau de bacharel e $0,2 \%$ o grau de doutor.

Quando questionados se possuíam formação especializada em enfermagem, a maioria dos participantes $(60,6 \%)$ respondeu negativamente. Dentre os que assinalaram possuir especialização, destaca-se a formação em enfermagem comunitária $(51,1 \%)$, seguindo-se enfermagem médico-cirúrgica $(17,4 \%)$ e enfermagem de reabilitação $(16,9 \%)$.
A percentagem mais expressiva de inquiridos trabalhava no setor público (59,3\%), seguindo-se os que exerciam a atividade principal no setor privado (37,9\%). No que respeita às áreas de exercício profissional da atividade principal, constatou-se que uma percentagem significativa a exercia na área hospitalar (41,3\%), na qual se destacaram, igualmente, os profissionais que exerciam atividade principal como enfermeiros do trabalho $(33,9 \%)$. A maioria possuía a categoria profissional de enfermeiro $(76,9 \%)$, seguindo-se a de enfermeiro especialista $(11,9 \%)$ e a de enfermeiro-chefe/responsável, com 7,8\%.

A principal motivação para trabalhar na área de Enfermagem do Trabalho era como complemento financeiro de outra atividade profissional $(42,4 \%)$. Seguiram-se os participantes que afirmaram ter sido, este campo de intervenção, a primeira opção (20,3\%). Trabalhavam como enfermeiros do trabalho 19,0\%, porque não conseguiram trabalhar noutra área. A maioria dos inquiridos $(88,1 \%)$ considerou ser pertinente a criação da área de especialização em Enfermagem do Trabalho. Destes, uma percentagem elevada $(85,1 \%)$ referiu que gostaria de frequentar a especialização em Enfermagem do Trabalho, se esta viesse a ser implementada.

Na Tabela 1, está presente a comparação da escala de perceção das áreas de conhecimento e intervenção do enfermeiro do trabalho (EPACIET) em função do género dos inquiridos. Nesta tabela, é possível constatar que as médias são sempre mais elevadas no género masculino, denotando-se a tendência para maior concordância com o facto de o enfermeiro do trabalho, na sua prática profissional, possuir conhecimentos e intervir em todas as áreas apresentadas. No entanto, as diferenças apenas eram assumidas como estatisticamente significativas em três áreas, mais propriamente na área Gestor $(\mathrm{t}=2,870 ; \mathrm{p}<0,01)$, Consultor $(\mathrm{t}=2,302 ; \mathrm{p}<0,05) \mathrm{e}$ na área Investigador $(\mathrm{t}=2,484 ; \mathrm{p}<0,05)$. Pelas médias, verificou-se que os elementos masculinos concordavam mais positivamente que o enfermeiro do trabalho devia ter conhecimentos e intervir nas áreas relacionadas com a gestão, consultadoria e investigação. 
Tabela 1 - Comparação das áreas da Escala de perceção das áreas de conhecimento e intervenção do enfermeiro do trabalho por género dos inquiridos. Portugal - 2017. (N=472)

\begin{tabular}{|c|c|c|c|c|c|}
\hline \multirow{2}{*}{ Variáveis } & \multirow{2}{*}{ Género } & \multirow{2}{*}{ Média } & \multirow{2}{*}{$\begin{array}{l}\text { Desvio } \\
\text { Padrão }\end{array}$} & \multicolumn{2}{|c|}{ Teste $t$} \\
\hline & & & & $t$ & $p$ \\
\hline \multirow[t]{2}{*}{ Clínico } & Masculino & 75,7 & 11,1 & 1,724 & 0,085 \\
\hline & Feminino & 73,5 & 12,6 & & \\
\hline \multirow[t]{2}{*}{ Especialista } & Masculino & 82,9 & 12,6 & 1,454 & 0,147 \\
\hline & Feminino & 80,9 & 13,1 & & \\
\hline \multirow[t]{2}{*}{ Gestor } & Masculino & 84,6 & 16,0 & 2,870 & 0,004 \\
\hline & Feminino & 79,7 & 16,5 & & \\
\hline \multirow[t]{2}{*}{ Coordenador } & Masculino & 82,9 & 17,1 & 1,477 & 0,140 \\
\hline & Feminino & 80,3 & 17,1 & & \\
\hline \multirow[t]{2}{*}{ Consultor } & Masculino & 82,1 & 19,2 & 2,302 & 0,022 \\
\hline & Feminino & 77,3 & 20,5 & & \\
\hline \multirow[t]{2}{*}{ Educador para a Saúde } & Masculino & 86,3 & 17,0 & 0,632 & 0,528 \\
\hline & Feminino & 85,2 & 17,3 & & \\
\hline \multirow[t]{2}{*}{ Conselheiro } & Masculino & 87,1 & 15,7 & 0,578 & 0,563 \\
\hline & Feminino & 86,2 & 15,4 & & \\
\hline \multirow[t]{2}{*}{ Investigador } & Masculino & 82,6 & 16,8 & 2,484 & 0,013 \\
\hline & Feminino & 77,7 & 19,6 & & \\
\hline
\end{tabular}

Fonte: Elaboração própria.

Os inquiridos que possuíam formação especializada privilegiaram as várias áreas identificadas. No entanto, as áreas de Clínico e Consetheiro foram as que obtiveram pontuação menos elevada. É possível verificar, na Tabela 2, que, nas áreas de conhecimento e intervenção do enfermeiro do trabalho relacionadas com a área Especialista, os inquiridos possuidores de formação especializada apresentaram a média mais elevada (Média=83,3; DP=11,1), e a diferença foi estatisticamente significativa ( $\mathrm{t}=2,653 ; p<0,01)$. O mesmo aconteceu para a área Gestor, em que os profissionais com formação especializada manifestaram a média mais alta (Média $=83,3$; $\mathrm{DP}=15,2$ ), e a diferença também foi estatisticamente significativa $(\mathrm{t}=2,524 ; p<0,05)$, o que pode estar relacionado com o facto de estes profissionais possuírem competências de gestão transversais às diferentes áreas de especialização em enfermagem.

Tabela 2 - Resultados das áreas da Escala de perceção das áreas de conhecimento e intervenção do enfermeiro do trabalho em função de formação especializada em enfermagem. Portugal - 2017. $(\mathrm{N}=472)$

\begin{tabular}{|c|c|c|c|c|c|}
\hline \multirow{2}{*}{ Variáveis } & \multirow{2}{*}{$\begin{array}{c}\text { Formação } \\
\text { especializada em } \\
\text { enfermagem }\end{array}$} & \multirow{2}{*}{ Média } & \multirow{2}{*}{$\begin{array}{l}\text { Desvio } \\
\text { Padrão }\end{array}$} & \multicolumn{2}{|c|}{ Teste $t$} \\
\hline & & & & $\boldsymbol{t}$ & $\boldsymbol{p}$ \\
\hline \multirow[t]{2}{*}{ Clínico } & Sim & 75,5 & 11,5 & 1,571 & 0,089 \\
\hline & Não & 73,2 & 12,6 & & \\
\hline \multirow[t]{2}{*}{ Especialista } & $\operatorname{Sim}$ & 83,3 & 11,1 & 2,653 & 0,008 \\
\hline & Não & 80,3 & 13,9 & & \\
\hline \multirow[t]{2}{*}{ Gestor } & Sim & 83,3 & 15,2 & 2,524 & 0,012 \\
\hline & Não & 79,5 & 17,1 & & \\
\hline \multirow[t]{2}{*}{ Coordenador } & Sim & 83,1 & 15,6 & 2,301 & 0,022 \\
\hline & Não & 79,6 & 17,9 & & \\
\hline \multirow[t]{2}{*}{ Consultor } & $\operatorname{Sim}$ & 82,7 & 17,8 & 3,781 & 0,000 \\
\hline & Não & 75,9 & 21,4 & & \\
\hline
\end{tabular}


Tabela 2 - Resultados das áreas da Escala de perceção das áreas de conhecimento e intervenção do enfermeiro do trabalho em função de formação especializada em enfermagem.

Portugal - 2017. (N=472)

(conclusão)

\begin{tabular}{l|c|c|c|c|c}
\hline \multirow{2}{*}{ Variáveis } & \multirow{2}{*}{$\begin{array}{c}\text { Formação } \\
\text { especializada em } \\
\text { enfermagem }\end{array}$} & Média & $\begin{array}{c}\text { Desvio } \\
\text { Padrão }\end{array}$ & \multicolumn{2}{|c}{ Teste t } \\
\cline { 5 - 6 } Educador para a Saúde & Sim & 88,9 & 15,2 & 3,621 & 0,000 \\
& Não & 83,3 & 18,1 & & \\
Conselheiro & Sim & 87,0 & 14,1 & 0,674 & 0,500 \\
& Não & 86,0 & 16,3 & & 0,000 \\
Investigador & Sim & 83,4 & 17,6 & 4,116 & \\
& Não & 76,2 & 19,3 & & \\
\hline
\end{tabular}

Fonte: Elaboração própria.

Relativamente à comparação dos resultados obtidos com o setor laboral no qual os inquiridos estavam inseridos (Tabela 3), constatou-se que, para todas as áreas da escala, não existiram diferenças estatisticamente significativas $(p>0,05)$.
Logo, pode depreender-se que o setor laboral no qual os inquiridos exerciam a atividade principal não tinha influência na sua opinião acerca das áreas de conhecimento e intervenção do enfermeiro do trabalho.

Tabela 3 - Resultados das áreas da Escala de perceção das áreas de conhecimento e intervenção do enfermeiro do trabalho por setor laboral da atividade principal. Portugal - 2017. (N=472)

\begin{tabular}{|c|c|c|c|c|c|}
\hline \multirow{2}{*}{ Variáveis } & \multirow{2}{*}{\begin{tabular}{|c|}
$\begin{array}{c}\text { Setor laboral (atividade } \\
\text { principal) }\end{array}$ \\
\end{tabular}} & \multirow{2}{*}{ Média } & \multirow{2}{*}{$\begin{array}{l}\text { Desvio } \\
\text { Padrão }\end{array}$} & \multicolumn{2}{|c|}{ Teste $t$} \\
\hline & & & & $t$ & $\boldsymbol{p}$ \\
\hline \multirow[t]{2}{*}{ Clínico } & Público & 74,7 & 11,7 & 1,254 & 0,210 \\
\hline & Privado & 73,2 & 13,0 & & \\
\hline \multirow[t]{2}{*}{ Especialista } & Público & 82,0 & 12,6 & 0,860 & 0,390 \\
\hline & Privado & 80,9 & 13,6 & & \\
\hline \multirow[t]{2}{*}{ Gestor } & Público & 81,1 & 16,8 & $-0,362$ & 0,717 \\
\hline & Privado & 81,7 & 15,6 & & \\
\hline \multirow[t]{2}{*}{ Coordenador } & Público & 81,4 & 17,3 & 0,261 & 0,794 \\
\hline & Privado & 81,0 & 16,3 & & \\
\hline \multirow[t]{2}{*}{ Consultor } & Público & 79,3 & 21,1 & 0,447 & 0,655 \\
\hline & Privado & 78,4 & 18,5 & & \\
\hline \multirow[t]{2}{*}{ Educador para a Saúde } & Público & 85,5 & 17,6 & $-0,567$ & 0,571 \\
\hline & Privado & 86,4 & 16,0 & & \\
\hline \multirow[t]{2}{*}{ Conselheiro } & Público & 87,1 & 14,8 & 0,815 & 0,416 \\
\hline & Privado & 85,9 & 15,9 & & \\
\hline \multirow[t]{2}{*}{ Investigador } & Público & 80,0 & 19,4 & 1,099 & 0,272 \\
\hline & Privado & 78,0 & 18,3 & & \\
\hline
\end{tabular}

Fonte: Elaboração própria.

Ao comparar as áreas de conhecimento e intervenção do enfermeiro do trabalho percecionadas com a variável exercício de funções como enfermeiro do trabalho (Tabela 4), observou-se que foi concretamente ao nível da área Investigador ( $\mathrm{t}=-2,523 ; p<0,05)$ que, pelas médias, aqueles que, no momento, não exerciam atividade enquanto enfermeiros do trabalho foram os que mais consideraram que este profissional podia intervir ao nível de Investigador (média mais elevada: Média=83,6; $\mathrm{DP}=18,2$ ). Os profissionais que exerciam, no momento, Enfermagem do Trabalho foram os que menos valorizaram esta área de conhecimento e intervenção. Nas demais áreas, não existiu diferença estatística dos resultados em função do exercício atual dos profissionais em Enfermagem do Trabalho $(p>0,05)$. 
Tabela 4 - Resultados das áreas da Escala de perceção das áreas de conhecimento e intervenção do enfermeiro do trabalho em função do exercício atual em Enfermagem do Trabalho. Portugal - 2017. $(\mathrm{N}=472)$

\begin{tabular}{|c|c|c|c|c|c|}
\hline \multirow[b]{2}{*}{ Variáveis } & \multirow{2}{*}{$\begin{array}{c}\text { Exerce atualmente } \\
\text { funções como enfermeiro } \\
\text { do trabalho? }\end{array}$} & \multirow[b]{2}{*}{ Média } & \multirow{2}{*}{$\begin{array}{l}\text { Desvio } \\
\text { Padrão }\end{array}$} & \multicolumn{2}{|c|}{ Teste $t$} \\
\hline & & & & $\boldsymbol{t}$ & $p$ \\
\hline \multirow[t]{2}{*}{ Clínico } & Sim & 74,0 & 12,3 & $-0,474$ & 0,636 \\
\hline & Não & 74,6 & 11,8 & & \\
\hline \multirow[t]{2}{*}{ Especialista } & Sim & 81,4 & 12,8 & $-0,186$ & 0,853 \\
\hline & Não & 81,7 & 13,7 & & \\
\hline \multirow[t]{2}{*}{ Gestor } & $\operatorname{Sim}$ & 80,7 & 16,4 & $-0,854$ & 0,393 \\
\hline & Não & 82,4 & 17,0 & & \\
\hline \multirow[t]{2}{*}{ Coordenador } & Sim & 80,7 & 17,0 & $-0,859$ & 0,391 \\
\hline & Não & 82,4 & 17,5 & & \\
\hline \multirow[t]{2}{*}{ Consultor } & Sim & 77,9 & 20,6 & $-1,558$ & 0,120 \\
\hline & Não & 81,6 & 18,5 & & \\
\hline \multirow[t]{2}{*}{ Educador para a Saúde } & $\operatorname{Sim}$ & 85,1 & 17,3 & $-0,935$ & 0,350 \\
\hline & Não & 87,0 & 17,0 & & \\
\hline \multirow[t]{2}{*}{ Conselheiro } & Sim & 86,5 & 15,2 & 0,266 & 0,790 \\
\hline & Não & 86,0 & 16,8 & & \\
\hline \multirow[t]{2}{*}{ Investigador } & Sim & 78,0 & 19,0 & $-2,523$ & 0,012 \\
\hline & Não & 83,6 & 18,2 & & \\
\hline
\end{tabular}

Fonte: Elaboração própria.

\section{Discussão}

Vale a pena salientar que, dos enfermeiros do trabalho inquiridos, relativamente ao futuro profissional, a maior parte afirmou qu gostaria de exercer a atividade como enfermeiro do trabalho em regime de tempo parcial. Por outro lado, uma percentagem menor afirmou que gostaria de trabalhar na Enfermagem do Trabalho em regime de tempo integral. A maioria dos inquiridos considerou ser pertinente a criação da área de especialização em Enfermagem do Trabalho.

Os resultados do presente estudo são extremamente expressivos. Da opinião dos enfermeiros do trabalho inquiridos, evidenciou-se o potencial da Enfermagem do Trabalho e do impacto que a sua atuação poderia ter no meio laboral. Eles expressaram uma opinião fortemente positiva face à pertinência da criação da especialização em Enfermagem do Trabalho em Portugal, justificando com a especificidade e complexidade do contexto de prestação de cuidados, e com a necessidade de valorização, visibilidade e reconhecimento desta área de intervenção. Ambicionavam formação ao nível de especialização em enfermagem, para a prestação de cuidados de Enfermagem do Trabalho de qualidade, com consequente aumento da autonomia funcional do enfermeiro do trabalho. Estes ansiavam por maior investimento na formação em Enfermagem do Trabalho, concretamente na formação considerada necessária, ao nível de especialidade, à semelhança das restantes áreas de especialização em enfermagem, tal como preconiza a Federação de Enfermeiros de Saúde Ocupacional da União Europeia ${ }^{(12)}$. Esta instituição considera que o desenvolvimento da enfermagem por diferentes áreas de especialização é fundamental para estimular o crescimento do conhecimento em enfermagem e para a melhoria da qualidade dos cuidados prestados à população ${ }^{(13)}$.

Da discussão acerca da formação para a enfermagem do trabalho, fica explícito que o referencial formativo afigura-se como uma estrutura de base válida, com as áreas nucleares essenciais, para integrarem uma proposta formativa para o enfermeiro do trabalho. Todavia, a promoção da saúde, a prevenção primária, secundária e terciária, e a avaliação do risco para a saúde e segurança dos trabalhadores no local de trabalho 
são áreas nucleares prioritárias a serem incluídas numa proposta formativa ${ }^{(14-15)}$.

Diante das competências do enfermeiro do trabalho, a formação necessária tem de ser consistente, específica e especializada, com um corpo de conhecimentos próprio, com vista à prestação de cuidados de qualidade em Enfermagem do Trabalho. Não existem dúvidas de que esta formação deve ser uma área de especialização em enfermagem, nomeadamente em Enfermagem do Trabalho.

Naturalmente que a conceção de uma proposta formativa para a Enfermagem do Trabalho, no que diz respeito à duração e metodologia, deve ser semelhante às formações existentes de especialização em enfermagem, atribuindo relevante importância à incorporação de estágios. Também fica claro que a proposta formativa, para dotar o enfermeiro do trabalho de conhecimentos específicos abrangentes, tem de ir ao encontro das áreas de conhecimento e intervenção, contribuindo para uma prática profissional de qualidade, bem como para a eficácia de atuação do enfermeiro do trabalho no seio da equipa de saúde ocupacional.

A proposta formativa deve ter como finalidade capacitar o enfermeiro para a aquisição de competências que permitam conceber, implementar e avaliar uma prática profissional em Enfermagem do Trabalho "especializada", tendo como suporte sustentado a evidência científica e a investigação.

Por meio das intervenções no âmbito da Enfermagem do Trabalho, em contexto de atuação multiprofissional, pretende-se que o profissional que atua nessa esfera seja capaz de assegurar, ao trabalhador ou grupo de trabalhadores no local de trabalho, o processo de cuidados de enfermagem focado na gestão da saúde e segurança do trabalhador na sua relação com o ambiente de trabalho, além de qualidade de vida e de trabalho ${ }^{(16-17)}$.

É preciso realçar que os elementos da amostra que fizeram parte deste estudo consideraram que o enfermeiro do trabalho deveria ter conhecimentos e intervir em todas as áreas identificadas, pois os valores estavam bastante acima do valor médio possível de obter (50 pontos). No entanto, foi possível constatar que há áreas de conhecimento e intervenção do enfermeiro do trabalho mais privilegiadas pelos inquiridos, que agora se elencam por ordem crescente, quanto à valorização: Conselheiro (Média=86,4), constituindo-se esta como a área mais privilegiada; Educador para a Saúde (Média=85,5); Especialista (Média=81,5); Gestor e Coordenador (Média=81,0). Apesar das médias serem consideráveis, as áreas que obtiveram os valores mais baixos, portanto consideradas menos privilegiadas pelos enfermeiros do trabalho, foram a área de Investigador (Média=79,0), Consultor (Média=78,6) e Clínico (Média=74,1).

\section{Conclusão}

Da análise do perfil sociodemográfico e socioprofissional dos enfermeiros do trabalho, é de mencionar que a desvalorização da Enfermagem do Trabalho, enquanto área de exercício, por parte dos enfermeiros do trabalho inquiridos, decorreu concretamente da sua motivação, pelo facto de maioritariamente exercerem-na como complemento financeiro à sua atividade principal ou por não terem conseguido colocação na área de exercício profissional da sua preferência.

Pôde-se concluir que o exercício profissional nas áreas de conhecimento e intervenção foi efetivamente desvalorizado ou não reconhecido e encarado pela maioria dos inquiridos como transitório, pelo facto de quase metade deles ambicionar trabalhar no futuro nesta área, mas apenas em regime de tempo parcial.

Fica demonstrado que é necessário e urgente maior investimento na formação em Enfermagem do Trabalho, concretamente a formação considerada necessária no nível da especialidade, à semelhança das restantes áreas de especialização em enfermagem.

\section{Colaborações:}

1 - concepção, projeto, análise e interpretação dos dados: Vitor Manuel Teixeira Machado, Maria João Filomena dos Santos Pinto Monteiro, 
Maria Helena de Oliveira Penaforte e Vitor Manuel Costa Pereira Rodrigues;

2 - redação do artigo e revisão crítica relevante do conteúdo intelectual: Vitor Manuel Teixeira Machado, Maria João Filomena dos Santos Pinto Monteiro e Maria Helena de Oliveira Penaforte;

3 - aprovação final da versão a ser publicada: Vitor Manuel Teixeira Machado, Maria João Filomena dos Santos Pinto Monteiro, Maria Helena de Oliveira Penaforte e Vitor Manuel Costa Pereira Rodrigues.

\section{Referências}

1. Agência Europeia para a Segurança e Saúde no Trabalho. Doenças relacionadas com o trabalho. [Internet]. Ljubljana (SVN); 2018 [cited 2020 Aug 21]. Available from: https://osha.europa.eu/ $\mathrm{pt} /$ themes/work-related-diseases

2. Almeida A, Santos M. A Enfermagem do Trabalho em Portugal: Experiência, Motivações, Locais de Trabalho, Formação, Funções, Indicadores produzidos e Condições de Trabalho. Rev Port Saúde Ocup on line. 2017;4:s1-s21. DOI: 10.31252/ RPSO.20.07.2017

3. American Association of Occupational Health Nurses. What is Occupational \& Environmental Health Nursing? [Internet]. Chicago (USA); 2012 [cited 2020 Aug 23]. Available from: http:// aaohn.org/page/profession-of-occupational-andenvironmental-health-nursing

4. Portugal. Decreto-Lei n. ${ }^{\circ} 71 / 2019$, de 27 de maio de 2019. Altera o regime da carreira especial de enfermagem, bem como o regime da carreira de enfermagem nas entidades públicas empresariais e nas parcerias em saúde [Internet]. Diário da República Eletrônico, n. 101, série I, de 27 de maio de 2019 [cited 2019 May 29]. Available from: https://dre.pt/dre/legislacao-consolidada/ decreto-lei/2019-122403273

5. Machado V, Martins A, Martins J, Ribeiro A, Santos S, Penaforte H. Áreas do conhecimento e de intervenção do enfermeiro do trabalho: Perspetiva dos enfermeiros. In: Moura C, Pereira I, Monteiro MJ, Pires P, Rodrigues V, coordenadores. Saúde: do Desafio ao Compromisso. Outeiro Seco (PT): Escola Superior de Enfermagem Dr. José Timóteo Montalvão Machado; 2015. p. 383-93.
6. Portugal. Direção-Geral da Saúde. Orientação 009/2014, de 3 de junho de 2014. Autorização para o exercício de Enfermagem do Trabalho [Internet]. Lisboa; 2014 [cited 2020 Sep 12]. Available from: https://www.sep.org.pt/files/ uploads/2016/09/2014_sep_dgs-utorizacao_para_ exercicio-enfermagem_no_trabalho.pdf

7. American Board for Occupational Health Nurses. The career guide to occupational health nursing [Internet]. Hinsdale (USA): 2014 [cited 2020 Sep 18]. Available from: https://pdf4pro.com/view/ career-guide-abohn-5a6216.html

8. Gomes J, Queirós P. Prática clínica do enfermeiro do trabalho. In: Borges E, coordenadora. Enfermagem do trabalho. Formação, investigação e estratégias de intervenção. Lisboa: Lidel; 2018. p. 67-80.

9. Teixeira A, Ferreira T, Borges E. Bullying no trabalho: Perceção e impacto na saúde mental e vida pessoal dos enfermeiros. RPESM. 2016;15:23-9. DOI: http://dx.doi.org/10.19131/rpesm.0128

10. Reknes I, Notelaers G, Magerøy N, Pallesen S, Bjorvatn B, Moen BE, et al. Aggression from Patients or Next of Kin and Exposure to Bullying Behaviors: A Conglomerate Experience? Nurs Res Pract. 2017:1502854. DOI: https://doi.org/10.1155/ 2017/1502854

11. Souza PMRA, Lopes ALSV, Hilal AVGH. Centralidade do trabalho na perspetiva de mulheres em diferentes faixas etárias. RACE. 2017;16(1):9-36. DOI: http://dx.doi.org/10.18593/ race.v16i1.13174

12. The Federation of Occupational Health Nurses within the European Union. Core Curriculum [Internet]. 3rd ed. Luxemburgo (LUX); 2014 [cited 2020 Sep 21]. Available from: https://fohneu.org/ images/pdf/CORE-CURRICULUM_2014.pdf

13. Oshodi TO, Crockett R, Bruneau B, West E. The nursing work environment and quality of care: A cross-sectional study using the Essentials of Magnetism II Scale in England. J Clin Nurs. 2017;26(17-18):2721-34. DOI: https://doi.org/10. 1111/jocn. 13783

14. Quintana Zavala MO, Valenzuela Suazo S, Paravic Klijn T. Enfermería desde la perspectiva del Trabajo Decente. Enferm Global. 2014 [cited 2018 Jul 22];13(33):310-6. Available from: http:// scielo.isciii.es/pdf/eg/v13n33/en_ensayo1.pdf

15. Wei H, Sewell KA, Woody G, Rose MA. The state of the science of nurse work environments 
in the United States: A systematic review. Int J Nurs Sci. 2018;5(3):287-300. DOI: https://doi. org/10.1016/j.ijnss.2018.04.010

16. Viselita F, Handiyani H, Pujasari H. Quality level of nursing work life and improvement interventions: Systematic review. Enferm Clin. 2019;29(Suppl 2):223-8. DOI: https://doi.org/10.1016/ j.enfcli.2019.06.001

17. Oshodi T, Bruneau B, Crockett R, Kinchington F, Nayar S, West E. The nursing work environment and quality of care: Content analysis of comments made by registered nurses responding to the Essentials of Magnetism II scale. Nurs Open. 2019;6(3):878-88. DOI: https://doi.org/10.1002/ nop 2.268

Recebido: 1 de agosto de 2021 Aprovado: 3 de novembro de 2021 Publicado: 7 de dezembro de 2021

A Revista Baiana de Enfermagem utiliza a Licença Creative Commons - Atribuição-NãoComercial 4.0 Internacional. https://creativecommons.org/licenses/by-nc/4.0/ Este artigo é de acesso aberto distribuído sob os termos da Licença Creative Commons (CC BY-NC). Esta licença permite que outros remixem, adaptem e criem a partir do seu trabalho para fins não comerciais. Embora os novos trabalhos tenham de lhe atribuir o devido crédito e não possam ser usados para fins comerciais, os usuários não têm de licenciar esses trabalhos derivados sob os mesmos termos. 